Marquette University

e-Publications@Marquette

Exercise Science Faculty Research and

Publications

Exercise Science, Department of

$3-2018$

\title{
Six-Minute Walk Test Performance in Persons With Multiple Sclerosis While Using Passive or Powered Ankle-Foot Orthoses
}

\author{
Morgan K. Boes \\ University of Illinois - Urbana-Champaign \\ Rachel Bollaert \\ Marquette University, rachel.bollaert@marquette.edu \\ Richard M. Kesler \\ Illinois Fire Service Institute \\ Yvonne C. Learmonth \\ Murdoch University \\ Mazharul Islam \\ University of Illinois - Urbana-Champaign
}

See next page for additional authors

Follow this and additional works at: https://epublications.marquette.edu/exsci_fac

\footnotetext{
Recommended Citation

Boes, Morgan K.; Bollaert, Rachel; Kesler, Richard M.; Learmonth, Yvonne C.; Islam, Mazharul; Petrucci, Matthew N.; Motl, Robert W.; and Hsiao-Wecksler, Elizabeth T., "Six-Minute Walk Test Performance in Persons With Multiple Sclerosis While Using Passive or Powered Ankle-Foot Orthoses" (2018). Exercise Science Faculty Research and Publications. 161.

https://epublications.marquette.edu/exsci_fac/161
} 
Authors

Morgan K. Boes, Rachel Bollaert, Richard M. Kesler, Yvonne C. Learmonth, Mazharul Islam, Matthew N.

Petrucci, Robert W. Motl, and Elizabeth T. Hsiao-Wecksler

This article is available at e-Publications@Marquette: https://epublications.marquette.edu/exsci_fac/161 
Marquette University

e-Publications@Marquette

\section{Exercise Science Faculty Research and Publications/College of Health Sciences}

This paper is NOT THE PUBLISHED VERSION; but the author's final, peer-reviewed manuscript. The published version may be accessed by following the link in th citation below.

Archives of Physical Medicine and Rehabilitation, Vol. 99, No. 3 (March 2018): 484-490. DOI. This article is (C Elsevier and permission has been granted for this version to appear in e-Publications@Marquette. Elsevier does not grant permission for this article to be further copied/distributed or hosted elsewhere without the express permission from Elsevier.

\section{Six-Minute Walk Test Performance in Persons With Multiple Sclerosis While Using Passive or Powered Ankle-Foot Orthoses}

Morgan K. Boes

Department of Bioengineering, University of Illinois at Urbana-Champaign, Champaign, IL

Rachel E. Bollaert

Department of Kinesiology and Community Health, University of Illinois at Urbana-Champaign, Champaign, IL

Richard M. Kesler

Illinois Fire Service Institute, Champaign, IL

Yvonne C. Learmonth

School of Psychology and Exercise Science, Murdoch University, Perth, Western Australia

Mazharul Islam

Department of Mechanical Science and Engineering, University of Illinois at Urbana-Champaign, Champaign, IL

Matthew N. Petrucci 
Department of Mechanical Science and Engineering, University of Illinois at Urbana-Champaign, Champaign, IL

Neuroscience Program, University of Illinois at Urbana-Champaign, Champaign, IL

Robert W. Motl

Department of Physical Therapy, The University of Alabama at Birmingham, Birmingham, AL

Elizabeth T. Hsiao-Wecksler

Department of Bioengineering, University of Illinois at Urbana-Champaign, Champaign, IL

Department of Mechanical Science and Engineering, University of Illinois at Urbana-Champaign, Champaign, IL

Neuroscience Program, University of Illinois at Urbana-Champaign, Champaign, IL

\section{Abstract}

Objective

To determine whether a powered ankle-foot orthosis (AFO) that provides dorsiflexor and plantar flexor assistance at the ankle can improve walking endurance of persons with multiple sclerosis (MS).

Design

Short-term intervention.

Setting

University research laboratory.

Participants

Participants $(\mathrm{N}=16)$ with a neurologist-confirmed diagnosis of $\mathrm{MS}$ and daily use of a prescribed custom unilateral passive AFO.

Interventions

Three 6-minute walk tests (6MWTs), 1 per footwear condition: shoes (no AFO), prescribed passive AFO, and portable powered AFO (PPAFO). Assistive devices were worn on the impaired limb.

\section{Main Outcome Measures}

Distance walked and metabolic cost of transport were recorded during each 6MWT and compared between footwear conditions.

\section{Results}

Each participant completed all three 6MWTs within the experimental design. PPAFO use resulted in a shorter 6MWT distance than did a passive AFO or shoe use. No differences were observed in metabolic cost of transport between footwear conditions.

\section{Conclusions}

The current embodiment of this PPAFO did not improve endurance walking performance during the 6MWT in a sample of participants with gait impairment due to MS. Further research is required to determine whether expanded training or modified design of this powered orthosis can be effective in improving endurance walking performance in persons with gait impairment due to MS. 


\section{Keywords}

Gait, Multiple sclerosis, Rehabilitation, Robotic exoskeleton,

\section{List of abbreviations}

6MWT, 6-minute walk test, AFO, ankle-foot orthosis, ANOVA, analysis of variance, CoT, metabolic cost of transport, MS, multiple sclerosis, PPAFO, portable powered ankle-foot orthosis, $\dot{\mathrm{V}} \mathrm{CO}_{2}$, carbon dioxide consumption per unit time, $\dot{\mathrm{V}}_{2}$, oxygen consumption per unit time

The major disease process of multiple sclerosis (MS) is characterized by demyelinating lesions of the white matter within the brain stem, cerebellum, and spinal cord. ${ }^{1}$ When these lesions occur along long-fiber tracts specific to lower limb muscle control, the clinical symptoms include weakness of the plantar flexor and

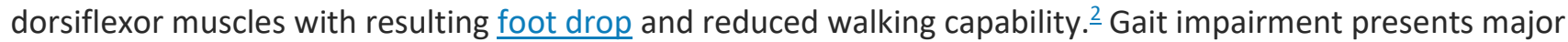

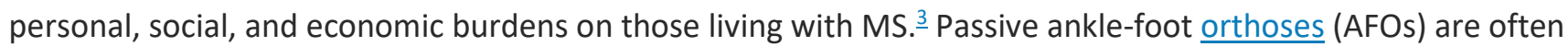
clinically prescribed and used to assist with foot drop due to dorsiflexor muscle weakness in persons with MS in attempts to mitigate gait impairment. ${ }^{4}$ AFOs are rigid or semirigid devices that support the foot and the lower leg to keep the foot from falling below a neutral position, thus preventing the foot drop that occurs with dorsiflexor muscle weakness. In holding the foot at neutral, most AFOs impede plantar flexion of the ankle and thus reduce the propulsive effect of the plantar flexor muscles during late stance.

Passive AFOs have yielded mixed results when analyzed in a research setting in populations of persons with MS $\underline{5}, \underline{6}, \underline{7}$ or MS and stroke. $\underline{8}, \underline{9}, \underline{10}$ Previous research has reported no significant improvement with an AFO compared to no-device trials on functional gait tasks,,-6 modified $\underline{6 \text {-minute walk test }}$ (6MWT) distance,,$\underline{7}$ or perceived fatigue during the $6 \mathrm{MWT}, \underline{\underline{I}}$ but there was reduced physiological cost of walking ${ }^{\underline{I}}$ and energy cost of walking. ${ }^{-}$Others 5,11 have reported that after 4 weeks of continually using an AFO, persons with MS reported fewer limitations in mobility (eg, walking, running, and stair climbing) when assessed using the Multiple Sclerosis Walking Scale-12. Some researchers have concluded that if the mechanical properties of the AFO (stiffness, neutral angle) matched the patient in terms of level of assistance needed, the patient greatly benefited with improved energy cost of walking, $\underline{\underline{12}}$ walking speed, gait kinematics, and kinetics. $\underline{\underline{8}}$ An AFO (constructed from an elastic band attached to the thigh and shank with a loop to hold up the forefoot) increased gait velocity and cadence, whereas mixed results were observed for stride length compared to a plastic-molded AFO and shoes condition. ${ }^{10}$ These studies fail to provide conclusive evidence that passive AFOs reliably improve gait in persons with MS.

Traditional AFOs often fail to restore normal ankle function because they lack the ability to actively modulate motion control during gait and do not produce propulsion torque and power. A test bed of a portable powered AFO (PPAFO) has been developed to explore the issues and challenges related to creating mobile actively powered orthotic devices.13, $\underline{14}$ One of the main objectives of the PPAFO test bed is to assist the ankle during gait. The PPAFO can provide modest dorsiflexor or plantar flexor torque at the ankle using a portable pneumatic power source (fig 1). The bidirectional assistance is applied at the ankle as needed throughout the gait cycle, including late stance and propulsion, which is unavailable in the current commercially available technologies. $\frac{15}{}$ The timing of when to apply the bidirectional assistance is determined by a user-specific tuned kinematics-based controller that estimates the state of the limb during the gait cycle using onboard sensors (foot switches, angle sensor).16, 17 Initial timings for PPAFO activation from the controller are based on normative gait event values determined from preexisting data $\underline{18}, \underline{19}$; subject-specific PPAFO actuation timing can then be adjusted for each user's gait pattern on the basis of variation from the normative sensor activation pattern. $\underline{17}$ 


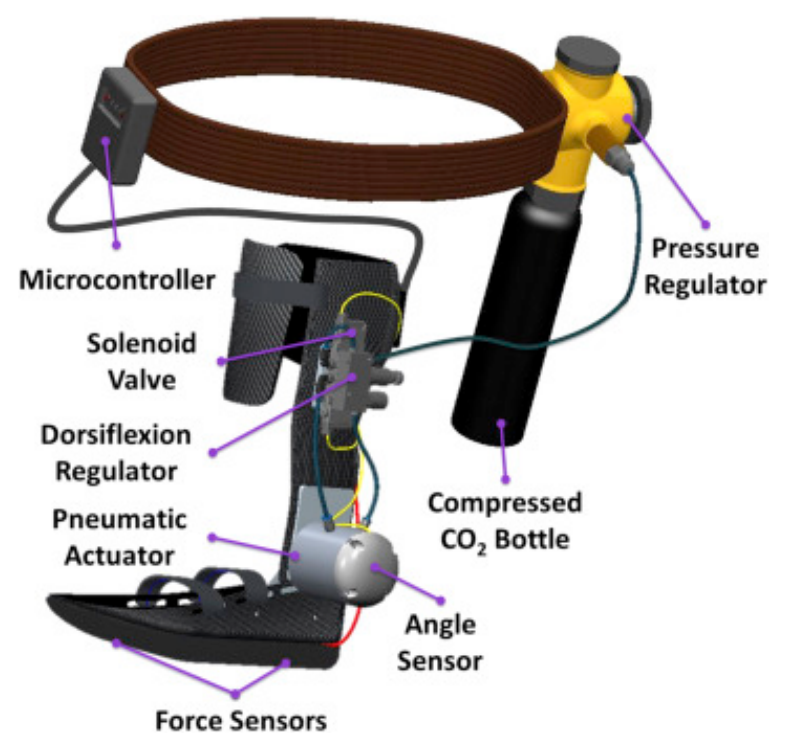

Fig 1. The PPAFO test bed. The PPAFO can give powered assistance in both dorsiflexion and plantar flexion directions.

Previous studies $13, \underline{20}$ using the PPAFO have tested the functionality of the concept in a limited number of people with lower limb impairment. One study $\underline{\underline{20}}$ tested the PPAFO in 2 participants with unique lower limb impairments ( 1 with muscular dystrophy, 1 with cauda equine syndrome) and provided only unilateral powered assistance in the direction of greatest weakness (plantar flexion or dorsiflexion) during treadmill walking. Another study $\underline{\underline{13}}$ further tested the PPAFO in 1 participant and provided bidirectional powered assistance that was triggered on the basis of direct user input. Since these initial studies, the PPAFO has undergone hardware and controller improvements that allow more diverse functionality testing.14, 16, 17, 21 The goal of the present investigation was to use a powered device that provided active bidirectional (dorsiflexor and plantar flexor) assistance at the ankle to improve the gait of persons with varied gait impairment due to MS. This study evaluated distance covered and metabolic cost in 6 minutes of walking with the PPAFO as compared with both a patient-prescribed AFO and a shoes-only condition during a 6MWT. It was hypothesized that the PPAFO would improve the gait of persons with MS by decreasing the metabolic cost of transport (COT) while increasing the 6MWT distance.

\section{Methods}

\section{Participants}

Approval for the study was granted by the institutional review board at the University of Illinois UrbanaChampaign, and all participants provided informed consent before the testing session. The study included 16 participants with a neurologist-confirmed diagnosis of MS and use of a prescribed custom passive AFO (12 women and 4 men; mean age, 54.6 $\pm 5.3 y$; median Expanded Disability Status Scale score, $\underline{22} 5.75$ [interquartile range, 4-6]); the patient reported MS type was as follows: primary progressive $(n=4)$, relapsing remitting $(n=7)$, and secondary progressive $(n=5)$. Assistive device use was as follows: single-point cane $(n=4)$, 2-wheeled walker $(n=1)$, 4-wheeled walker ( $n=3)$, walls and arms of caregivers $(n=2)$, and none $(n=6)$.

\section{Experimental procedure}

Participants first wore the PPAFO for a 20-minute training period, which was for both the participant to accommodate to walking with the PPAFO and for each participant's gait pattern to be programmed into the PPAFO controller using sensor data from the heel, toe, and angle sensors on the PPAFO to estimate the phases 
of gait. Participants were instructed to walk in a normal way with the PPAFO. For each participant, controller timing adjustments were made throughout the training period to best align the participant's sensor activation pattern with the phases of gait and correlated assistance timing. $\underline{17}^{17}$

Participants then completed three 6MWTs that were performed overground in a $9 \mathrm{~m} \times 12 \mathrm{~m}$ hallway loop with four $90^{\circ}$ turns. For each $6 \mathrm{MWT}$, participants were instructed to walk as far and as fast as possible within the limits of their own safety. $\frac{23}{}$ One 6MWT was completed per footwear condition: shoes, AFO, and PPAFO. A minimum rest period of 10 minutes was provided to all participants between each 6MWT. $\underline{24}$ The PPAFO was worn on the more affected limb (ie, the same side as the prescribed AFO) with the participant's normal walking shoe on the contralateral limb. The electronics control box of the PPAFO was worn at the chest on an over-theshoulder harness, and a research assistant following the participant carried the portable pneumatic air tank. The 6MWT with the shoe-only condition was completed first as a baseline for all participants. The remaining 2 conditions (AFO, PPAFO) were randomized and counterbalanced in order across participants ( $\underline{\text { fig } 2}$ ).

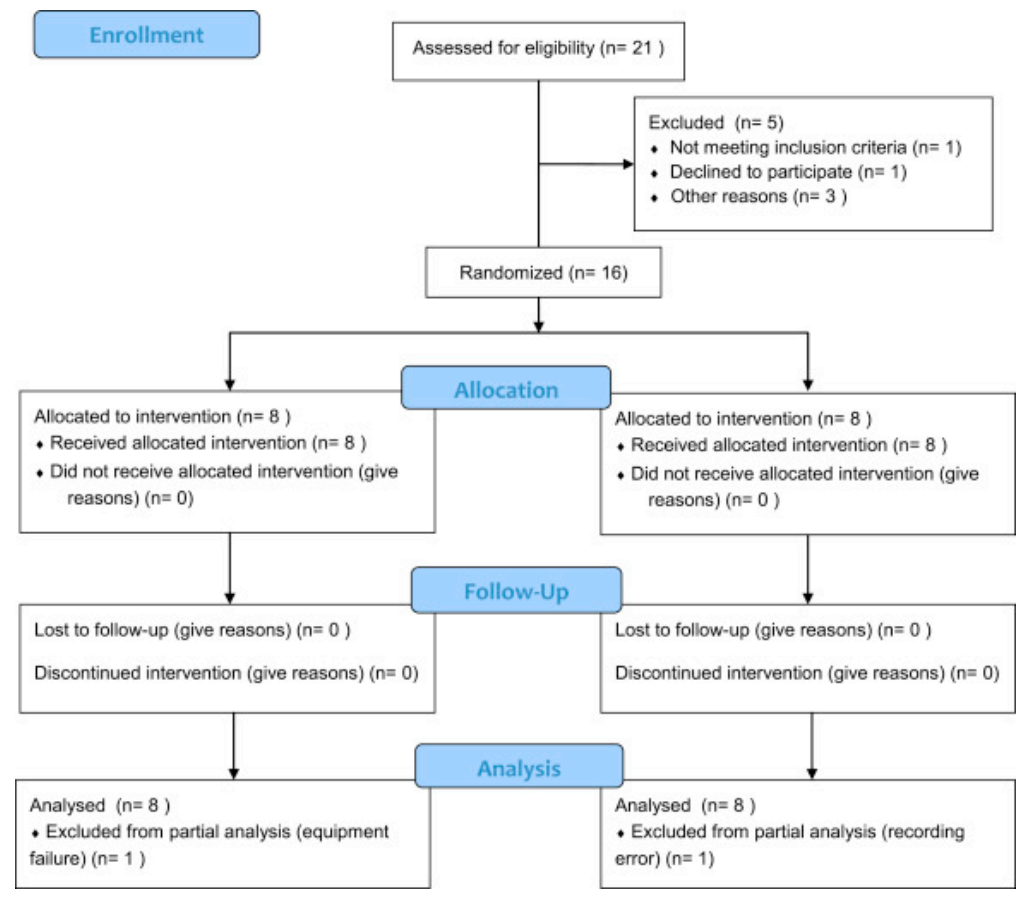

Fig 2. CONSORT diagram.

\section{Outcome measures}

Total distance traveled and CoT were recorded during each 6MWT. The total 6MWT distance traveled (in meters) was recorded with a measuring wheel (RT312a). Oxygen consumption per unit time $\left(\dot{\mathrm{V}}_{2}, \mathrm{~mL} / \mathrm{min}\right)$ and carbon dioxide consumption per unit time $\left(\dot{\mathrm{V}}_{\mathrm{CO}}, \mathrm{mL} / \mathrm{min}\right)$ were measured breath by breath using a commercially available portable metabolic unit (K4b2- $)$. $\dot{V} \mathrm{O}_{2}$ and $\dot{\mathrm{V}} \mathrm{CO}_{2}$ were measured as 30-second averages for 1 minute before the 6MWT (resting) and during the 6MWT (walking). Steady-state values were computed from average walking values of the final 3 minutes of the $6 \mathrm{MWT} . \underline{25}$ Net values were computed by subtracting average resting values from the steady-state values (Eqns. $\underline{1}$ and $\underline{2}$ ).

(1) $\left[\dot{\mathrm{V}} \mathrm{o}_{2}\right]_{\text {net }}(\mathrm{mL} / \mathrm{min})=\left[\dot{\mathrm{V}} \mathrm{o}_{2}\right]_{\text {steady-state }}-\left[\dot{\mathrm{V}} \mathrm{o}_{2}\right]_{\text {resting }}$

(2) $\left[\dot{\mathrm{V}} \mathrm{CO}_{2}\right]_{\text {net }}(\mathrm{mL} / \mathrm{min})=\left[\dot{\mathrm{V}} \mathrm{CO}_{2}\right]_{\text {steady-state }}-\left[\dot{\mathrm{V}} \mathrm{CO}_{2}\right]_{\text {resting }}$ 
CoT was used to quantify the energy expenditure during gait. $\underline{26}$ CoT was computed from the net $\dot{\mathrm{V}}_{2}$ and $\dot{\mathrm{V}} \mathrm{CO}_{2}$ using a modification to the Brockway equation ${ }^{27}$ as suggested by Donelan et al, ${ }^{26}$ with updated coefficients (Eqn. $\underline{3}) . \underline{28}$

(3) Metabolic Cost of Transport (CoT) $=$

$\frac{16.477(\mathrm{~W} \cdot \mathrm{s} / \mathrm{mL}) \times\left[\dot{\mathrm{V}}_{2}\right]_{\text {net }}(\mathrm{mL} / \mathrm{min})+4.484(\mathrm{~W} \cdot \mathrm{s} / \mathrm{mL}) \times\left[\dot{\mathrm{V}} \mathrm{co}_{2}\right]_{\text {net }}(\mathrm{mL} / \mathrm{min})}{60(\mathrm{~s} / \mathrm{min}) \times \text { tody }}$ $60(\mathrm{~s} / \mathrm{min}) \times$ body weight $(\mathrm{N}) \times$ gait speed $(\mathrm{m} / \mathrm{s})$

For the PPAFO condition, the additional mass of the PPAFO and controller box ( $2 \mathrm{~kg})$ was added to the participant's mass to be included in the body weight.

Statistical analysis

6MWT distance and metabolic data were analyzed for the effect of footwear. A repeated-measures analysis of variance (ANOVA) was completed for each parameter, with footwear as the within-subject factor. Because of equipment and tester error, 1 participant completed the 6MWTs but the final distance was not available; another participant completed the 6MWTs but there was an error with the portable metabolic unit; so each parameter was computed for the remaining 15 participants. All statistical tests were performed (SPSS version $\left.22^{\circ}\right)$ with the level of significance set at .05 .

\section{Results}

All 16 participants completed the three 6MWTs in the shoes, AFO, and PPAFO conditions without injury. Each participant donned and doffed the PPAFO with ease. Each participant practiced walking with the PPAFO as it was trained to their gait until they felt comfortable enough to complete a 6MWT, and this was achieved during the 20-minute training period.

\section{MWT distance}

The repeated-measures ANOVA for 6MWT distance indicated significant differences between footwear $(P=.003)$. The follow-up least significant difference comparisons between footwear conditions indicated that PPAFO use resulted in a shorter 6MWT distance than did AFO and shoe use. Also, AFO use resulted in a longer 6MWT distance than did shoe-only use ( $\underline{\text { fig } 3}$ and table 1$)$.

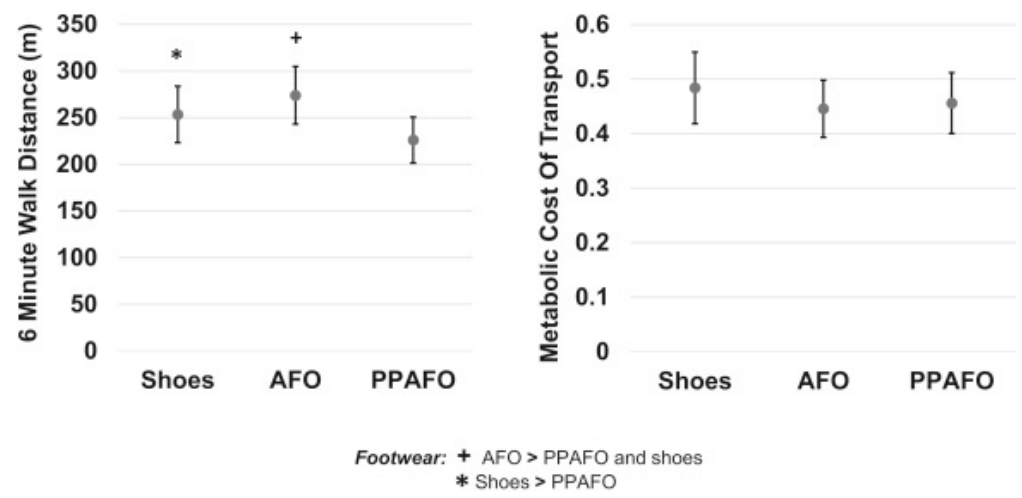

Fig 3. CoT and 6MWT distance for 15 persons with MS in 3 footwear conditions: shoes, passive AFO, or PPAFO. Values are mean $\pm \mathrm{SE}$. Symbols represent significant differences $(P<.05)$.

Table 1. Outcome measure values

\begin{tabular}{|l|l|l|l|l|}
\hline Variable & Shoes & AFO & PPAFO & $\boldsymbol{P}$ \\
\hline 6MWT distance $(\mathrm{m})$ & $253.3 \pm 30.2^{*}$ & $273.8 \pm 31.0^{ \pm}$ & $225.9 \pm 24.7$ & .003 \\
\hline CoT & $0.48 \pm 0.07$ & $0.45 \pm 0.05$ & $0.46 \pm 0.06$ & .23 \\
\hline
\end{tabular}


NOTE. Values are mean \pm SE.

$*$ Shoes $>$ PPAFO.

†AFO $>$ PPAFO and shoes.

\section{Metabolic outcomes}

The repeated-measures ANOVA for CoT indicated no significant differences $(P=.23)$ between footwear conditions (see fig 3 and table 1).

\section{Discussion}

This study was the first to assess the use of a unilateral powered AFO to provide bidirectional powered ankle assistance for persons with MS by evaluating the total distance covered in 6 minutes of walking and the CoT. Each participant successfully donned the PPAFO for an overground 6MWT during which the PPAFO was programmed to assist with their gait. Participants completed three 6MWTs, 1 with the PPAFO, 1 with their own passive AFO, and 1 with only shoes. During each 6MWT, total distance covered and metabolic parameters were recorded.

The use of the PPAFO significantly reduced the 6MWT distance covered by participants in our study. This was in contrast to our expected outcome of increasing the 6MWT distance with the use of a powered orthosis at least compared to a shoes-only condition. One possibility is that in our participant population, disability from MS had already limited their mobility such that learning to walk with a new device could not improve their performance. It is also possible that participants were becoming accustomed to using the PPAFO in their gait, but it was at the expense of the total distance covered in the 6MWT. Participants may have benefited from a longer training period to adapt to walking with the PPAFO. $\underline{29}, \underline{30}$

No differences were observed in CoT between footwear conditions. It was hypothesized that the PPAFO condition would reduce the COT in comparison to the shoes and AFO conditions because of the PPAFO being able to assist each participant with the symptoms of foot drop and reduced propulsion. The lack of expected significant reduction in CoT with the PPAFO could have been due to the increased mass of the PPAFO at the ankle that took increased energy to walk with, thus confounding any reduction in CoT that may have been due to the PPAFO's assistance. $31, \underline{32} \mathrm{~A}$ more extended time to adapt to and become comfortable with walking with the PPAFO may be necessary to realize gait changes that would allow a reduction in CoT, especially in persons with MS whose adaptation abilities could be impaired by nervous system damage. 29 , $\underline{30}$ Other studies $\underline{33}, \underline{34}, \underline{35}, \underline{36}, \underline{37}$ using powered exoskeletons to assist or augment gait in able-bodied persons have rarely shown the expected decrease in metabolic effort. It is possible that there are underlying factors that have yet to be determined to make a meaningful effect on reducing the metabolic cost of walking.

The resulting increase in 6MWT distance with the use of an AFO compared with shoes seen in this study is in line with previous research. In agreement with previous studies, the passive AFO footwear condition resulted in faster walking speeds (greater 6MWT distance) than did a shoes-only condition; however, the previously reported reduction in metabolic parameters of walking due to wearing an AFO was not observed (see fig 3). $\underline{7}, \underline{8}, \underline{9}$ The lack of this difference could be due to a number of factors, such as a difference in the participants' use of their AFOs or different calculations of metabolic parameters. $\underline{12}, \underline{22}, \underline{23}$, $\underline{38}$ Future studies of AFO use in persons with gait impairment should quantify the amount of daily use that a participant uses their AFO, how many years they have used an AFO, and possibly what type or types of AFOs the person has used as confounding factors. The only inclusion criteria for this study were that a participant had an AFO. Simply having an AFO does not necessarily mean that each of the participants used their AFOs in similar ways or for similar amounts of time.12, 27, 28, 34 It is expected that the amount of metabolic benefit that one would receive from using an AFO is related to the amount when the AFO is actually used. 


\section{Study limitations}

The shorter 6MWT distance observed when wearing the PPAFO could be due to a number of factors. Participants were given a minimum of 20 minutes to adapt to the device because the controller was programmed to their gait while wearing the PPAFO. A possible alternative controller programming strategy could have improved participant performance with the PPAFO. $\underline{39}$ Also, even more training and practice could provide time to improve gait function. Some studies have suggested that anywhere from 20 minutes to multiple days of use may be necessary to adapt to a powered ankle orthosis $\underline{40}, \underline{41}, \underline{42}, \underline{43}$ and upward of 4 weeks may be necessary to adapt to a passive AFO. $\frac{5}{}$

Some of the participant's gait might have been affected by the fit and comfort of the PPAFO devices. Even though the devices were sized and fit to the participant, they were not custom fabricated for each participant. Custom therapeutic AFOs range in weight based on the type and size; for example, for a medium-sized female participant, a carbon fiber strut would weigh $0.1 \mathrm{~kg}$, a solid thermoplastic AFO would weigh $\sim 0.4 \mathrm{~kg}$, and a thermoplastic hybrid with metal ankle joints and uprights would be upward of $\geq 0.7 \mathrm{~kg}$. The PPAFO added weight to the distal ankle (total PPAFO weight, 1.8kg), possibly affecting the participant's gait. 31 , 32 The PPAFO was also designed with a heel rocker and forefoot rocker in the sole that were not accounted for in the gait analysis completed here. $\underline{44}, \underline{45}, \underline{46}$

\section{Conclusions}

This study was the first to test a powered AFO in a population of persons with neurological disability, specifically MS. The PPAFO was fitted and programmed to 16 persons with MS, and each completed a 6MWT using the PPAFO. Participants also completed a 6MWT with their own AFO and in a shoes only (no device) condition. The successful completion of a 6MWT with the PPAFO in persons with varied disability levels due to MS is an achievement toward developing a device with bidirectional powered ankle torque for gait assistance.

The 6MWT distance and CoT computed in this study indicate that within this study design, participants did not overcome their gait impairment while using the PPAFO. Yet, the PPAFO did not negatively affect the CoT used to walk. The lack of gait improvement with the PPAFO could be due to any number of factors, such as a need for more training and experience walking with the PPAFO, fatigue, or a need for improved device design. The PPAFO has additional areas for further development, such as more advanced controls. $\underline{17}, \underline{39} \mathrm{~A}$ hardware redesign for the PPAFO would be beneficial to further reduce weight and improve the comfort of the device while increasing the amount of assistive torque that it is able to produce. Along with primary gait assistance, the PPAFO test bed could be used to further understand biomechanical adaptation in persons with MS, especially regarding gait and gait therapies. Because the disease process of MS includes ever-changing and progressive symptoms, this actively changing physiology allows a unique opportunity to also continue to use the PPAFO test bed to develop robotic devices that respond naturally and automatically to the changing needs of the user.

Suppliers
a. RT312; Rolatape.
b. K4b2; COSMED Srl.
c. SPSS version 22; IBM Inc.

\section{Acknowledgments}

This work was made possible by help from Ziming Wang, MS, and undergraduates in the Human Dynamics and Controls Laboratory in the Department of Mechanical Science and Engineering. We thank Gavin Horn, PhD, and the Illinois Fire Service Institute for the use of their facilities. 


\section{References}

1 J.H. Noseworthy, C. Lucchinetti, M. Rodriguez, B.G. Weinshenker Multiple sclerosis N Engl J Med, 343 (2000), pp. 938-952

$\underline{2}$ R.W. Motl Ambulation and multiple sclerosis Phys Med Rehabil Clin North Am, 24 (2013), pp. 325-336

$\underline{3}$ J. Pike, E. Jones, K. Rajagopalan, J. Piercy, P. Anderson Social and economic burden of walking and mobility problems in multiple sclerosis BMC Neurol, 12 (2012), pp. 1-8

4 J. Wening, J. Ford, L.D. Jouett Orthotics and FES for maintenance of walking in patients with MS Dis Mon, 59 (2013), pp. 284-289

$\underline{5}$ G.M. Ramdharry, J.F. Marsden, B.L. Day, A.J. Thompson De-stabilizing and training effects of foot orthoses in multiple sclerosis Mult Scler J, 12 (2006), pp. 219-226

$\underline{6}$ L.R. Sheffler, M.T. Hennessey, J.S. Knutson, G.G. Naples, J. Chae Functional effect of an ankle foot orthosis on gait in multiple sclerosis: a pilot study Am J Phys Med Rehabil, 87 (2008), pp. 26-32

7 J.V. McLoughlin, S.R. Lord, C.J. Barr, M. Crotty, D.L. Sturnieks Dorsiflexion assist orthosis reduces the physiological cost and mitigates deterioration in strength and balance associated with walking in people with multiple sclerosis Arch Phys Med Rehabil, 96 (2015), pp. 226-232.e31

$\underline{8}$ D.J. Bregman, V. de Groot, P. Van Diggele, H. Meulman, H. Houdijk, J. Harlaar Polypropylene ankle foot orthoses to overcome drop-foot gait in central neurological patients: a mechanical and functional evaluation Prosthet Orthot Int, 34 (2010), pp. 293-304

$\underline{9}$ D.J. Bregman, J. Harlaar, C.G. Meskers, V. de Groot Spring-like ankle foot orthoses reduce the energy cost of walking by taking over ankle work Gait Posture, 35 (2012), pp. 148-153

10 Y.I. Hwang, W.G. Yoo, D.H. An, H.J. Heo The effect of an AFO-shaped elastic band on drop-foot gait in patients with central neurological lesions NeuroRehabilitation, 32 (2013), pp. 377-383

11 J.C. Hobart, A. Riazi, D.L. Lamping, R. Fitzpatrick, A.J. Thompson Measuring the impact of MS on walking ability-the 12-Item MS Walking Scale (MSWS-12) Neurology, 60 (2003), pp. 31-36

12 M.A. Brehm, F. Nollet, J. Harlaar Energy demands of walking in persons with postpoliomyelitis syndrome: relationship with muscle strength and reproducibility Arch Phys Med Rehabil, 87 (2006), pp. 136-140

13 K.A. Shorter, G.F. Kogler, E. Loth, W.K. Durfee, E.T. Hsiao-Wecksler A portable-powered-ankle-foot-orthosis for rehabilitation J Rehabil Res Dev, 48 (2011), pp. 459-472

14 Boes MK, Islam M, Li YD, Hsiao-Wecksler ET. Fuel efficiency of a portable powered ankle-foot orthosis. In: IEEE International Conference on Rehabilitation Robotics; 2013 Jun 24-26; Seattle (WA).

15 K.A. Shorter, J.C. Xia, E.T. Hsiao-Wecksler, W.K. Durfee, G.F. Kogler Technologies for powered ankle-foot orthotic systems: possibilities and challenges IEEE/ASME Trans Mechatron, 18 (2013), pp. 337-347

16 D.Y. Li, A. Becker, K.A. Shorter, T. Bretl, E.T. Hsiao-Wecksler Estimating system state during human walking with a powered ankle-foot orthosis IEEE/ASME Trans Mechatron, 16 (2011), pp. 835-844

17 M. Islam, M.T. Hagan, E.T. Hsiao-Wecksler Gait state estimation for a powered ankle orthosis using modified fractional timing and artificial neural network J Med Devices, 10 (2016), p. 020920

$\underline{18}$ J. Perry Gait analysis: normal and pathological function Slack, Thorofare (1992)

19 C. Kirtley Introduction: clinical gait analysis Churchill Livingstone, Edinburgh (2006), pp. 201-222

20 K.A. Shorter, Y. Li, E.A. Morris, G.F. Kogler, E.T. Hsiao-Wecksler Experimental evaluation of a portable powered ankle-foot orthosis Conf Proc IEEE Eng Med Biol Soc, 2011 (2011), pp. 624-627

$\underline{21}$ M. Islam, E.T. Hsiao-Wecksler Detection of gait modes using an artificial neural network during walking with a powered ankle-foot orthosis J Biophys, 2016 (2016), p. 7984157

$\underline{22}$ J.F. Kurtzke Rating neurologic impairment in multiple sclerosis: an Expanded Disability Status Scale (EDSS) Neurology, 33 (1983), pp. 1444-1452

23 M.D. Goldman, R.A. Marrie, J.A. Cohen Evaluation of the six-minute walk in multiple sclerosis subjects and healthy controls Mult Scler J, 14 (2008), pp. 383-390 
24 B.M. Sandroff, R.W. Motl, L.A. Pilutti, et al. Accuracy of StepWatch and ActiGraph accelerometers for measuring steps taken among persons with multiple sclerosis PLoS One, 9 (2014), p. e93511

$\underline{25}$ R.W. Motl, E.M. Snook, S. Agiovlasitis, Y. Suh Calibration of accelerometer output for ambulatory adults with multiple sclerosis Arch Phys Med Rehabil, 90 (2009), pp. 1778-1784

26 J.M. Donelan, R. Kramand, A.D. Kuo Mechanical and metabolic determinants of the preferred step width in human walking Proc R Soc B Biol Sci, 268 (2001), pp. 1985-1992

27 J.M. Brockway Derivation of formulae used to calculate energy expenditure in man Hum Nutr Clin Nutr, 41 (1987), pp. 463-471

$\underline{28}$ P.G. Adamczyk, S.H. Collins, A.D. Kuo The advantages of a rolling foot in human walking J Exp Biol, 209 (2006), pp. 3953-3963

$\underline{29}$ V. Tomassini, P.M. Matthews, A.J. Thompson, et al. Neuroplasticity and functional recovery in multiple sclerosis Nat Rev Neurol, 8 (2012), pp. 635-646

30 D.J. Ksiazek-Winiarek, P. Szpakowski, A. Glabinski Neural plasticity in multiple sclerosis: the functional and molecular background Neural Plast, 2015 (2015), p. 307175

$\underline{31}$ S.L. Barnett, A.M. Bagley, H.B. Skinner Ankle weight effect on gait: orthotic implications Orthopedics, 16 (1993), pp. 1127-1131

32 R.C. Browning, J.R. Modica, R. Kram, A. Goswami The effects of adding mass to the legs on the energetics and biomechanics of walking Med Sci Sports Exer, 39 (2007), pp. 515-525

33 L.M. Mooney, E.J. Rouse, H.M. Herr Autonomous exoskeleton reduces metabolic cost of human walking J Neuroeng Rehabil, 11 (2014), p. 151

34 L.M. Mooney, E.J. Rouse, H.M. Herr Autonomous exoskeleton reduces metabolic cost of human walking during load carriage J Neuroeng Rehabil, 11 (2014), p. 80

35 P. Malcolm, W. Derave, S. Galle, D. De Clercq A simple exoskeleton that assists plantarflexion can reduce the metabolic cost of human walking PLoS One, 8 (2013), p. e56137

$\underline{6} 6$ G.S. Sawicki, D.P. Ferris Powered ankle exoskeletons reveal the metabolic cost of plantar flexor mechanical work during walking with longer steps at constant step frequency J Exp Biol, 212 (2009), pp. 21-31

37 C.J. Walsh, K. Endo, H. Herr A quasi-passive leg exoskeleton for load-carrying augmentation Int J Human Robot, 4 (2007), pp. 487-506

$\underline{38}$ L.A. Pilutti, D. Dlugonski, B.M. Sandroff, et al. Gait and six-minute walk performance in persons with multiple sclerosis J Neurol Sci, 334 (2013), pp. 72-76

39 M. Islam, E.T. Hsiao-Wecksler Developing a classification algorithm for plantarflexor actuation timing of a powered ankle-foot orthosis J Med Devices, 10 (2016)

40 S.M. Cain, K.E. Gordon, D.P. Ferris Locomotor adaptation to a powered ankle-foot orthosis depends on control method J Neuroeng Rehabil, 4 (2007), p. 48

$\underline{41}$ K.E. Gordon, D.P. Ferris Learning to walk with a robotic ankle exoskeleton J Biomech, 40 (2007), pp. 26362644

$\underline{42}$ G.S. Sawicki, D.P. Ferris Mechanics and energetics of level walking with powered ankle exoskeletons J Exp Biol, 211 (2008), pp. 1402-1413

$\underline{43}$ Norris JA, Marsh AP, Granata KP, Ross SD. Positive feedback in powered exoskeletons: improved metabolic efficiency at the cost of reduced stability? In: ASME 2007 International Design Engineering Technical Conferences and Computers and Information in Engineering Conference; 2007 Jan 1; Las Vegas (NV).

44 S. Hutchins, P. Bowker, N. Geary, J. Richards The biomechanics and clinical efficacy of footwear adapted with rocker profiles-evidence in the literature Foot, 19 (2009), pp. 165-170

45 J.T. Long, J.P. Klein, N.M. Sirota, J.J. Wertsch, D. Janisse, G.F. Harris Biomechanics of the double rocker sole shoe: gait kinematics and kinetics J Biomech, 40 (2007), pp. 2882-2890

46 K.A. Myers, J.T. Long, J.P. Klein, J.J. Wertsch, D. Janisse, G.F. Harris Biomechanical implications of the negative heel rocker sole shoe: gait kinematics and kinetics Gait Posture, 24 (2006), pp. 323-330 
Supported by the National Science Foundation Engineering Research Center for Compact and Efficient Fluid Power (award no. 0540834) and the Foundation of the Consortium of Multiple Sclerosis Centers' MS Workforce of the Future program.

Disclosures: M.K. Boes reports grants and personal fees from the National Science Foundation and grants from the Foundation of the Consortium of Multiple Sclerosis Centers (during the conduct of the study). M. Islam reports grants from the National Science Foundation Engineering Research Center for Compact and Efficient Fluid Power (outside the submitted work). M.N. Petrucci reports grants and personal fees from National Science Foundation (during the conduct of the study). R.W. Motl reports grants from the Consortium of Multiple Sclerosis Centers (during the conduct of the study). E.T. Hsiao-Wecksler reports grants from National Science Foundation (during the conduct of the study); in addition, E.T. Hsiao-Wecksler is a holder of a U.S. patent (patent no. US9480618 B2). The other authors have nothing to disclose. 